Tropical Journal of Pharmaceutical Research October 2019; 18 (10): 2117-2123

ISSN: $1596-5996$ (print); 1596-9827 (electronic)

(C) Pharmacotherapy Group, Faculty of Pharmacy, University of Benin, Benin City, 300001 Nigeria.

Available online at http://www.tjpr.org

http://dx.doi.org/10.4314/tjpr.v18i10.17

Original Research Article

\title{
Effect of "Xiaoke Tongbi granule" on the proliferation, migration and tubule-forming ability of rat endothelial progenitor cells under high glucose conditions
}

\author{
Youcai Yuan ${ }^{1}$, Xiaoke Zhang ${ }^{1}$, Huan Zhang ${ }^{2}$, Bifeng Gao ${ }^{3}$ \\ ${ }^{1}$ Shaanxi University of Traditional Chinese Medicine, Xianyang, ${ }^{2}$ Chengdu University of Traditional Chinese Medicine, \\ Chengdu, Sichuan Province, ${ }^{3}$ Affiliated Hospital of Shaanxi University of Traditional Chinese Medicine, Xianyang, Shaanxi \\ Province, China
}

*For correspondence: Email: mt96ro@163.com

Sent for review: 7 August 2019

Revised accepted: 30 September 2019

\begin{abstract}
Purpose: To investigate the effect of Xiaoke Tongbi granule (XTG) on the proliferation, migration and tubule-forming ability of endothelial progenitor cells (EPCs) of rats under high glucose conditions.

Methods: Six specific pathogen-free (SPF) and twenty-four healthy rats (mean weight $=200 \pm 20 \mathrm{~g}$ ) were used in this study. Twenty-four (24) healthy rats were treated with graded concentrations of XTG $(0.75-2.25 \mathrm{~g} / \mathrm{mL})$ for 7 days, and were thereafter euthanized to obtain serum which was later used to treat EPCs isolated from bone marrow of SPF rats. The EPCs were seeded in culture plates pre-coated with human fibronectin, and cultured at $37{ }^{\circ} \mathrm{C}$ for $72 \mathrm{~h}$ in a humidified atmosphere of $5 \% \mathrm{CO}_{2}$ and $95 \%$ air. Cell viability and apoptosis were assessed using 3 (4,5 dimethyl thiazol 2 yl) 2,5 diphenyl $2 \mathrm{H}$ tetrazolium bromide (MTT), and flow cytometric assays, respectively. The morphology of isolated EPCs was assessed by immunofluorescence.

Results: The isolated EPCs exhibited normal morphology, and were CD34-positive. Proliferation and migration of EPCs, and number of tubular structures formed were significantly suppressed under high glucose conditions, but were significantly and concentration-dependently promoted by XTG treatment ( $p$ $<0.05)$. Treatment with XTG also significantly improved the morphology of isolated EPCs $(p<0.05)$. Apoptosis was significantly promoted by high glucose conditions, but was significantly and concentration-dependently reduced by XTG treatment $(p<0.05)$. The incidence of tubule formation in high glucose group was $0.63 \%$, but was progressively increased from 1.37 to $1.52 \%$ after treatment with graded concentrations of XTG.

Conclusion: These results indicate that XTG reverses the effect of high glucose environment on EPC proliferation, migration and tubule-forming ability.
\end{abstract}

Keywords: High glucose conditions, Xiaoke Tongbi granule, Endothelial progenitor cells, Proliferation, Tubule formation

This is an Open Access article that uses a fund-ing model which does not charge readers or their institutions for access and distributed under the terms of the Creative Commons Attribution License (http://creativecommons.org/licenses/by/4.0) and the Budapest Open Access Initiative (http://www.budapestopenaccessinitiative.org/read), which permit unrestricted use, distribution, and reproduction in any medium, provided the original work is properly credited.

Tropical Journal of Pharmaceutical Research is indexed by Science Citation Index (SciSearch), Scopus, International Pharmaceutical Abstract, Chemical Abstracts, Embase, Index Copernicus, EBSCO, African Index Medicus, JournalSeek, Journal Citation Reports/Science Edition, Directory of Open Access Journals (DOAJ), African Journal Online, Bioline International, Open-J-Gate and Pharmacy Abstracts

\section{INTRODUCTION}

The cellular and molecular processes that control vascular injury responses after percutaneous coronary intervention involve a complex interplay between vascular cells and progenitor cells, a process which controls arterial remodeling, neointimal proliferation, and re-re- 
endothelialization. The re-endothelialization of injured vascular endothelium and angiogenesis of the ischemic lesion play important roles in the repair of vascular injury caused by diabetes mellitus (DM) and cardiovascular diseases (CVDs). Diabetes mellitus- and CVD-induced damages to endothelial cells are restored by two processes: endothelial repair and angiogenesis $[1,2]$.

Xiaoke Tongbi granule (XTG) is used in Traditional Chinese Medicine (TCM) to facilitate the migration and homing of EPCs to ischemic muscle tissues and nerves, thereby contributing significantly to angiogenesis. It also facilitates blood supply to injured limbs via the upregulation of vascular endothelial growth factor (VEGF), hypoxia-inducible factor 1 (HIF-1) and PI3K/Akt protein expressions in ischemic limb muscles, thereby reinforcing the angiogenic effect. The present study investigated the effects of XTG on the proliferation, migration and tubule-forming ability of EPCs under high glucose conditions.

\section{EXPERIMENTAL}

\section{Drugs and reagents}

Xiaoke Tongbi granule (XTG) was obtained from the Preparation Center of the Affiliated Hospital of Shaanxi University of TCM. Fetal bovine serum (FBS), M199 medium and trypsin solution were products of Gibco (USA). Vascular endothelial growth factor (VEGF), fibroblast growth factor (FGF) and epithelial cell growth factor (EGF) were obtained from Peprotech (USA), while EBM-2 high glucose medium was purchased from Invitrogen (USA). Annexin VFITC apoptosis kit and CD34-FITC monoclonal antibody were products of $\mathrm{BD}$ Biosciences (USA); FLK-1-PE monoclonal antibody was purchased from Sigma-Aldrich (USA), while phosphate-buffered saline (PBS) and MTT assay kit were products of Solarbio (China).

This research was approved by the Animal Ethical Committee of Affiliated Hospital of Shaanxi University of Traditional Chinese Medicine, Xianyang, Shaanxi Province, China (approval no. 201822142), and performed according to "Principles of Laboratory Animal Care" (NIH publication no. 85-23, revised 1985) [3].

\section{Animals}

Six SPF and twenty-four healthy rats (mean weight, $200 \pm 20 \mathrm{~g}$ ) were purchased from the Animal Experimental Center of Xi'an Jiaotong University School of Medicine (No. scxk (Shaan)
2013-003). The ratio of male to female rats was 50:50.

\section{Rat grouping and treatment}

Twenty-four healthy rats were randomly assigned to four groups of six rats each: control group, $0.75 \mathrm{~g} / \mathrm{mL}$ XTG group, $1.5 \mathrm{~g} / \mathrm{mL}$ XTG group, and $2.25 \mathrm{~g} / \mathrm{mL}$ XTG group. XTG was administered orally using gavage. The control group rats received $2 \mathrm{~mL}$ of normal saline consecutively for 7 days. At the end of the treatment period, the rats were anesthetized with pentobarbital sodium, and blood was collected through the abdominal aorta $1 \mathrm{~h}$ after the last administration. After $24 \mathrm{~h}$ incubation at $4{ }^{\circ} \mathrm{C}$, the blood was centrifuged at $3000 \mathrm{rpm}$ for $15 \mathrm{~min}$ to obtain serum. The serum was sterilized using $0.22 \mu \mathrm{m}$ microporous membrane filter, and refrigerated at $-20^{\circ} \mathrm{C}$ until use.

\section{Isolation and culture of EPCs}

The SPF rats were anesthetized with $10 \%$ chloral hydrate intraperitoneally at a dose of 0.35 $\mathrm{mL} / 100 \mathrm{~g}$. Then, rat knee joint and distal femur were excised and exposed, and two holes were thereafter drilled in them using a dental drill. Two syringes were separately attached: one was used to inject sterile PBS, and the other to extract bone marrow. The extracted bone marrow was dispersed into individual cells by subjecting it to density gradient centrifugation at $1500 \mathrm{rpm}$ for $15 \mathrm{~min}$ at $4{ }^{\circ} \mathrm{C}$ using lymphocyte separation solution (a mixture of Ficoll and sodium diatrizoate) as the density gradient solution.

The bone marrow cells were subsequently resuspended in M199 medium containing $10 \mathrm{ng} / \mathrm{mL}$ VEGF, $10 \%$ FBS, $5 \mathrm{ng} / \mathrm{mL} F G F$ and $5 \mathrm{ng} / \mathrm{mL}$ EGF. The resultant cell suspension was seeded in culture plates pre-coated with human fibronectin, and cultured at $37{ }^{\circ} \mathrm{C}$ for $72 \mathrm{~h}$ in a humidified atmosphere of $5 \% \mathrm{CO}_{2}$ and $95 \%$ air. The medium was changed every 2 days. The cells were observed under an inverted microscope, and those in logarithmic growth phase were selected and used in subsequent experiments.

\section{Identification of EPCs}

The isolated EPCs were stimulated with rhodamine-labeled CD34 antibody and incubated overnight at $4{ }^{\circ} \mathrm{C}$. Then, 4', 6-diamidino-2phenylindole (DAPI) was used for nuclear staining, and the EPCs were photographed using an inverted fluorescence microscope. Positive cells produced red fluorescence with blue nuclei. 


\section{Grouping of EPCs and treatment}

The EPCs were randomly assigned to five groups: normal control group (C group), high glucose control group (G group), Z1 group, Z2 group and Z3 group. The cells were pretreated with phosphatidylinositide-3-kinase (PI3K) inhibitor, Wortmannin $(0.2 \mu \mathrm{mol} / \mathrm{L})$ or endothelial nitric oxide synthase (eNOS) inhibitor, L-NAME $(200 \mu \mathrm{mol} / \mathrm{L})$ for $30 \mathrm{~min}$. Cells in $\mathrm{C}$ group were cultured in EBM-2 complete medium only, while in $G$ group the medium was replenished with EBM-2 high glucose medium containing 30 $\mathrm{mmol} / \mathrm{L}$ glucose. Cells in Z1 group were cultured in EBM-2 high glucose medium supplemented with 15-fold concentration of serum XTG ZT; Z2 group cells were cultured in EBM-2 high glucose medium supplemented with 10 -fold concentration of serum XTG; and cells in Z3 group were cultured in EBM-2 high glucose medium containing 5 times the concentration of serum XTG. Each group of cells was cultured at $37^{\circ} \mathrm{C}$. Morphological changes in the cells were assessed at intervals of 18,36 and $72 \mathrm{~h}$.

\section{MTT assay}

The proliferative ability of EPCs in the presence of XTG was assessed using MTT assay. The cells were seeded at a density of $1 \times 10^{6}$ cells/well in 96-well plates and cultured in Dulbecco's modified Eagle's medium (DMEM) for 24 h. Then, serum containing graded concentrations of XTG $(0.75-2.25 \mathrm{~g} / \mathrm{mL})$ was added to the cells and incubated for $72 \mathrm{~h}$. At the end of the third day, $20 \mu \mathrm{L}$ of MTT solution (5 $\mathrm{mg} / \mathrm{mL}$ ) was added to the wells, followed by incubation at $37{ }^{\circ} \mathrm{C}$ for $2 \mathrm{~h}$. The medium was finally replaced with $150 \mu \mathrm{L}$ of $0.1 \%$ dimethyl sulfoxide (DMSO) to completely dissolve the formazan crystals formed. The absorbance of the samples was read in a microplate reader at 490 $\mathrm{nm}$. The assay was performed in triplicate. Cell proliferation $(\mathrm{P})$ was calculated as shown in Eq 1.

$\mathrm{P}(\%)=(\mathrm{Ae} / \mathrm{Ac}) 100$

Where $\mathrm{Ae}$ and $\mathrm{Ac}$ are the absorbance of experimental and control rats.

\section{Apoptosis assay}

The EPCs were seeded at a density of $2.5 \times 10^{6}$ cells/well in 6-well plates and cultured for $24 \mathrm{~h}$. Then, serum containing graded concentrations of XTG $(0.75-2.25 \mathrm{~g} / \mathrm{mL})$ was added to the medium and incubated for another $72 \mathrm{~h}$. Thereafter it was washed with PBS, and thoroughly mixed with $200 \mu \mathrm{L}$ binding buffer. The cells were then stained with $5 \mu \mathrm{L}$ each of annexin V-fluorescein isothiocyanate and propidium iodide within $25 \mathrm{~min}$ at room temperature in the dark. Cell apoptosis was assessed using a flow cytometer fitted with argon laser operated at $485 \mathrm{~nm}$.

\section{In vitro cell migration and invasion assay}

The cells $\left(1 \times 10^{4}\right.$ cells/well $)$ were placed in Transwell chamber coated with substrate and cultured in serum-free medium. Medium containing $10 \%$ FBS was added to the lower chamber. After $24 \mathrm{~h}$, the cells that passed through the matrix gel membrane were stained with crystal violet after fixation with $4 \%$ paraformaldehyde for $10 \mathrm{~min}$, photographed and counted using an inverted microscope.

\section{Lumen formation assay}

The cells were seeded in 24-well plates (at a density of $\times 10^{7}$ cells $/ \mathrm{mL}$, and $300 \mu \mathrm{L}$ of matrigel was added to each well, followed by culturing at $37{ }^{\circ} \mathrm{C}$ for $60 \mathrm{~min}$. Thereafter, graded concentrations of XTG in serum were added. After culturing for $20 \mathrm{~h}$, tubular arrangement, and the number and integrity of tubular structures were examined under an inverted microscope using an Image-ProPlus 5.1 software so as to analyze the total tubule structure per field $(\times 40)$. The results were expressed as percentages relative to the control.

\section{Statistical analysis}

Data are expressed as mean \pm SEM, and statistical analysis was performed using SPSS (version 19.0). Groups were compared using Student $t$-test. Values of $p<0.05$ were considered statistically significant.

\section{RESULTS}

\section{Vascular EPC morphology}

As shown in Figure 1, the isolated EPCs exhibited normal morphology. The shape was fusiform with a small number of dendrites. An increase in cell density led to cell-to-cell interaction and cross-linking.

\section{Expression of CD34 in vascular EPCs}

Results of immunofluorescence staining showed that the cells were highly purified. They were CD34-positive, with red fluorescence and blue nuclei (Figure 2). 


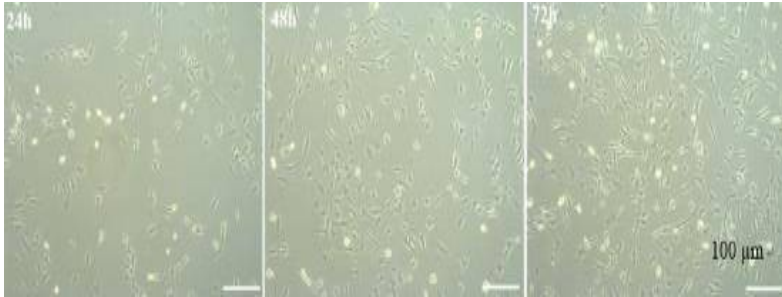

Figure 1: Morphology of vascular EPCs after 24, 48 and $72 \mathrm{~h}$ of culture

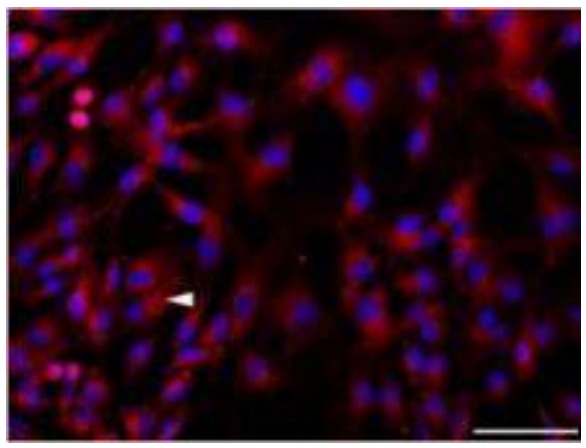

Figure 2: Immunofluorescence analysis of vascular EPCs

\section{Effect of XTG on morphology and proliferation of isolated EPCs in high glucose conditions}

The proliferation of EPCs was significantly suppressed under high glucose condition, but this suppression was significantly and concentration-dependently reversed by XTG treatment $(p<0.05)$. Treatment with XTG also significantly improved the morphology of isolated EPCs $(p<0.05)$. These results are shown in Figure 3, Figure 4 and Figure 5.

\section{Effect of XTG on apoptosis of EPCs}

Apoptosis was significantly promoted by high glucose condition, but was significantly and concentration-dependently reduced by XTG treatment $(p<0.05$; Figure 6$)$.

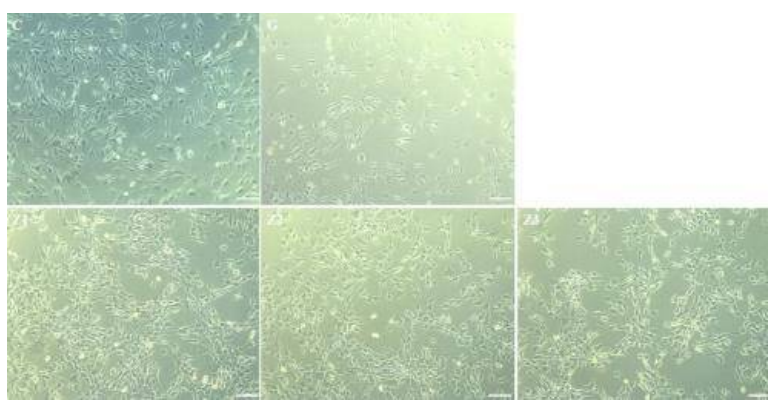

Figure 3: Effect of XTG on morphology of EPCs

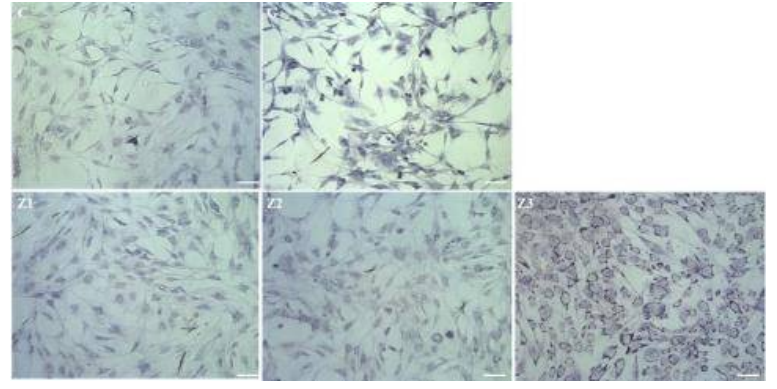

Figure 4: Changes in the morphology of EPCs after treatment with $\mathrm{XTG}$ as revealed by DAPI staining

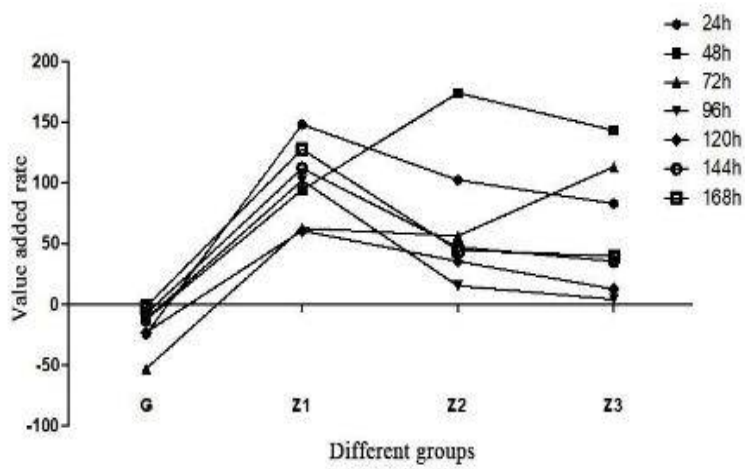

Figure 5: MTT assay results

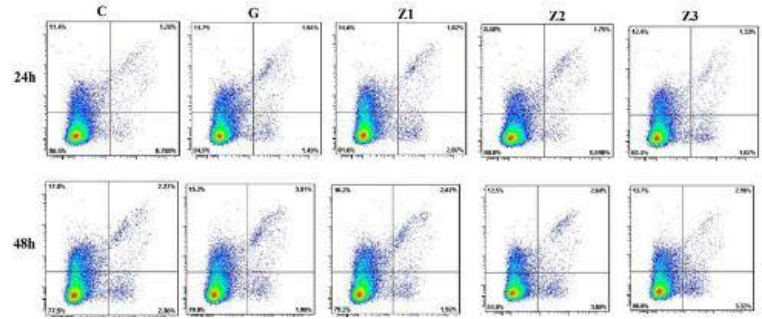

Figure 6: Flow cytometric results for EPCs after treatment with XTG

As shown in Figure 7, the migratory ability of EPCs was significantly reduced under condition of high glucose, but was significantly and concentration-dependently increased after treatment with XTG $(p<0.05)$.

\section{Effect of XTG on extracellular lumen formation}

Vascular endothelial cells in the control group were able to form distinct tubular structures. The number of tubular structures was significantly reduced under high glucose condition, but this was significantly and concentration-dependently increased after treatment with XTG $(p<0.05)$. The incidence of tubule formation in high glucose group was $0.63 \%$, but was progressively increased from 1.37 to $1.52 \%$ after treatment with graded concentrations of XTG (Figure 8). 

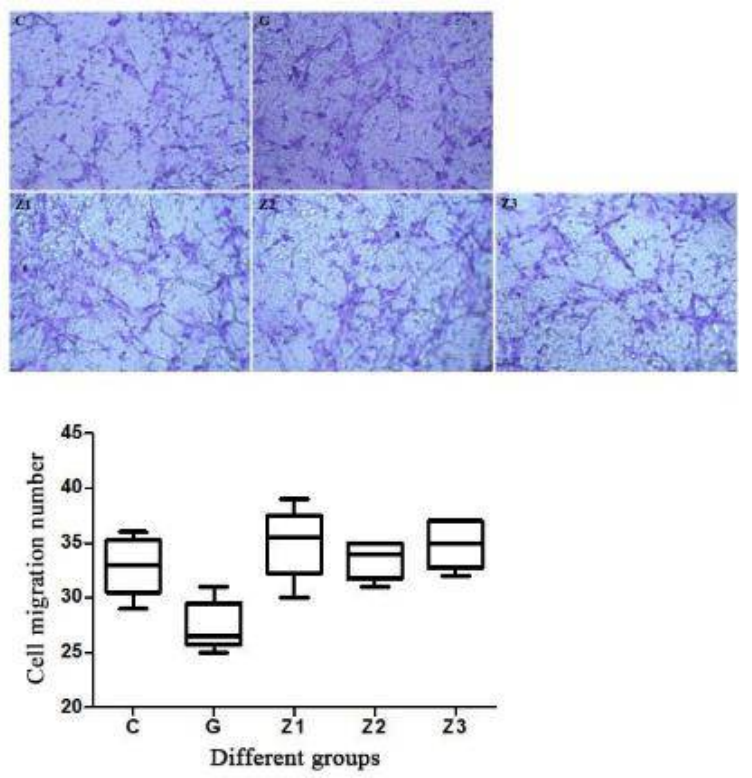

Figure 7: Effect of XTG on cell migration, obtained from Transwell assay

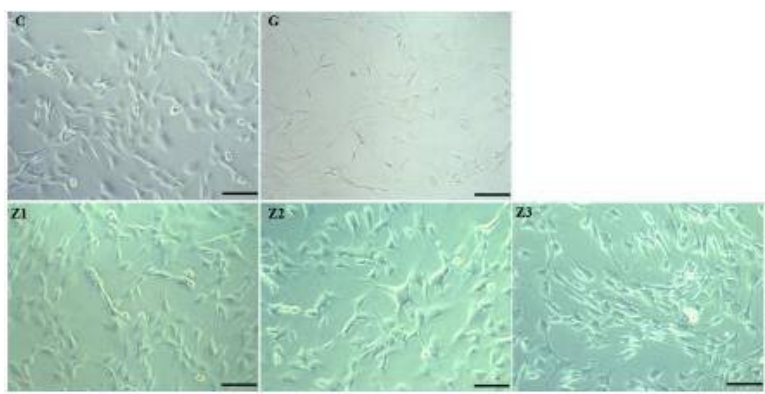

Figure 8: Effect of XTG on tubule-forming ability of EPCs

\section{DISCUSSION}

Diabetic macro- and microvascular complications result in vascular endothelial injury, accompanied by impaired or decreased number of EPCs. Thus, a possible way to treat vascular dysfunction and injury is by promoting the circulation of EPCs to regions of vascular injury and stimulating their differentiation into mature endothelial cells. In diabetic vascular disease, EPCs from different sources induce angiogenesis and indirectly reinforce neovascularization in ischemic tissues through division, proliferation and migration of the cells. Neovascularization stimulates the formation of functional microvascular networks capable of perfusion by red blood cells which serve as collateral circulation in response to local poor perfusion or ischemia. This enhances nerve function by promoting nerve regeneration and intraneural angiogenesis, thereby facilitating axonal extension, and promoting the survival of neurons, as well as satellite and Schwann cells [4-6].

Peripheral neuropathy (PN), a common complication of DM, refers to nerve damage caused by hyperglycemia. It is characterized by numbness, loss of sensation, and sometimes pain in the feet, legs, or hands. An understanding of its pathogenesis helps to unravel the relationship between fasting blood glucose variability and coronary artery collateral formation [7]. In TCM, the concept of "pulse and collateral - vascular system disease" is aimed at explaining the therapeutic effect of "replenishing $q i$ and generating pulse" and "activating blood and generating pulse". This provides basis for treatment of vascular lesions in TCM.

The results of this study indicate that XTG may promote qi, activate blood circulation and remove stasis, thereby facilitating recovery from vascular endothelial cell damage caused by diseases such as atherosclerosis, DM and peripheral vascular diseases [8].

Astragalus and Panax notoginseng accelerate the proliferation, migration and differentiation of EPCs, and promote endothelial cell repair under hyperglycemic conditions [9]. The bioactive compounds in Angelica, Salvia and Pueraria have been shown to activate blood circulation, removes stasis, and promote the proliferation, migration, adhesion and angiogenesis of EPCs, thereby reinforcing the repair of damaged endothelial cells and angiogenesis, both in vitro and in vivo [10-12]. A specially formulated drug for the treatment of diabetic PN, XTG consists of Astragalus, Pueraria, Angelica, Mulberry white, Clematis and Daphne giraldii Nitsche. It replenishes qi and activates blood circulation, while dredging collaterals and relieving pain. Studies have shown that when properly used, XTG accelerates the migration and homing of EPCs to ischemic muscle tissues and nerves in diabetic ischemic conditions; stimulates angiogenesis, and facilitates blood supply to the affected limbs. Vascular endothelial growth factor (VEGF) and HIF - 1 are highly expressed in peripheral nerves and blood vessels at the site of ischemic lesions, and they promote local endothelial cell proliferation, angiogenesis, and blood supply [13-17].

In this study, the proliferation and migration of EPCs, and number of tubular structures formed were significantly suppressed under high glucose medium, but were significantly and concentration-dependently promoted by XTG treatment. Treatment with XTG also significantly 
improved the morphology of isolated EPCs. Apoptosis was significantly promoted by condition of high glucose, but was significantly and concentration-dependently reduced by XTG treatment. It is likely that the mechanism underlying the observed effects of XTG involves increased expression of PI3K/Akt/eNOS signal pathway-related proteins.

\section{CONCLUSION}

The results of this study suggest that XTG reverses the effects of high glucose condition on EPC proliferation, migration and tubule-forming ability.

\section{DECLARATIONS}

\section{Acknowledgement}

This work was supported with grants from the National Natural Science Foundation of China (81273750) and National Natural Science Foundation of Shaanxi Province (no. 2012jM4016).

\section{Conflict of interest}

No conflict of interest is associated with this work.

\section{Contribution of authors}

We declare that this work was done by the author(s) named in this article and all liabilities pertaining to claims relating to the content of this article will be borne by the authors. All authors read and approved the manuscript for publication. Bifeng Gao conceived and designed the study, Youcai Yuan, Xiaoke Zhang, Huan Zhang, Bifeng Gao collected and analysed the data, while Youcai Yuan and Xiaoke Zhang wrote the manuscript.

\section{Open Access}

This is an Open Access article that uses a funding model which does not charge readers or their institutions for access and distributed under the terms of the Creative Commons Attribution License (http://creativecommons.org/licenses/by/ 4.0) and the Budapest Open Access Initiative (http://www.budapestopenaccessinitiative.org/rea d), which permit unrestricted use, distribution, and reproduction in any medium, provided the original work is properly credited.

\section{REFERENCES}

1. Olaniran KO, Eneanya ND, Allegretti AS, Zhao $S H$, Achebe MM, Thadhani RI. Cardiovascular Outcomes in African Americans with Sickle Cell Trait and Chronic Kidney Disease. Am J Nephrol 2019; 49(2): 93-102.

2. Jialal I, Fadini GP, Pollock $K$. Circulating levels of endothelial progenitor cell mobilizing factors in the metabolic syndrome. Cardiol 2010; 106(11): 1606 1608.

3. World Health Organization. Principles of laboratory animal care. WHO Chron 1985; 39: 51-56.

4. Zhao $T$, Li J, Chen AF. Micro RNA-34a induces endothelial progenitor cell senescence and impedes its angiogenesis via suppressing silent information regulator 1. Am J Physiol Endocrinol Metab 2010; 299(1): 110 - 116.

5. Wang $C$, Deng $Y$, Yue $Y$, Chen $W$, Zhang $Y$, Shi $G, W u$ Z. Glutamine Enhances the Hypoglycemic Effect of Insulin in L6 Cells via Phosphatidylinositol-3-Kinase (PI3K)/Protein Kinase B (AKT)/Glucose Transporter 4 (GLUT4) Signaling Pathway. Med Sci Monit 2018; 24: 1241-1250.

6. Lee JH, Jung S, Park WS, Choe EK, Kim E, Shin R, Heo SC, Lee JH, Kim K, Chai YJ. Prognostic nomogram of hypoxia-related genes predicting overall survival of colorectal cancer-Analysis of TCGA database. Sci Rep 2019; 9: 1803.

7. Liu CP, Liu ZY, Liu JP, Kang Y, Mao CS, Shang J. Diagnostic Value of Common Inflammatory Markers on Fever of Unknown Origin. Jpn J Infect Dis 2016; 69(5): 378-383.

8. Wu YL, Wei C, Jia ZH. Outline of Pulse and Merdian Theory and Its Application. J Tradit Chin Med 2014; 55(3): 181 - 184.

9. Shen L, Yang BH, Zeng JJ. Effects of Astragalus and Notoginseng on the differentiation of endothelial progenitor cells in high glucose environment. Beijing Tradit Chin Med 2010; 29(10): 787 - 789.

10. Ye RY, Chen JS. Effect of salvia miltiorrhiza injection on the number and activity of endothelial progenitor cells in patients with cerebral infarction. J Zhejiang Tradit Chin Med 2011; 46(2): 153.

11. Qin Z, Huang SQ. Effect of Danggui Buxue Decoction on endothelial progenitor cells of rabbit atherosclerosis and serum VEGF, SDF-1. J Chin Pathophysiol 2012; 28(2): 211-215.

12. Gao D, Jiao YH, Wu YM. Experimental Study on Xuefu Zhuyu Decoction Inducing Endothelial Progenitor Cells to Participate in Angiogenesis in the Ischemic Area. Chin J Integr Tradit West Med 2012; 2(5): 224-228.

13. Zhang XK, Chen WM, Lei M. Effect of Xiaoke Tongbi Granule on Neovascularization and Neural Structure of Rats with Ischemic Peripheral Neuropathy under Hyperglycemia. Tradit Chin Pharm Med 2011; 33(11): 1972-1975

14. Zhang XK, Yuan YC, Deng C. Effect of Xiaoketongbi Granule on the Expression of HIF-1a and VEGF in

Trop J Pharm Res, October 2019; 18(10):2122 
Ischemic Muscle of Diabetic Rats. Mod J Integr Tradit Chin West Med 2016; 25(17): 1825 - 1827.

15. Yu N, Fang $X$, Zhao $D, M u Q$, Zuo J, Ma $Y$, Zhang $Y$, Mo $F$, Zhang $D$, Jiang $G$, et al. Anti-Diabetic Effects of Jiang Tang Xiao Ke Granule via PI3K/Akt Signalling Pathway in Type 2 Diabetes KKAy Mice. Plos One 2017; 12(1): 168980.

16. Xu X, Liu F, Zhang S, Jia J, Li Z, Guo X, Yang Y, Sun H, You $Q$. Indenoisoquinoline derivatives as topoisomerase
I inhibitors that suppress angiogenesis by affecting the HIF signaling pathway. Biomed Pharmacother 2013; 67(8): 715-722.

17. Tan GH, Liu YY, Wang L, Li K, Zhang ZQ, Li HF, Yang $Z F$, Li Y, Li D, Wu MY, et al. PRRT2 deficiency induces paroxysmal kinesigenic dyskinesia by regulating synaptic transmission in cerebellum. Cell Res 2018; 28(1): 90-110. 